Western University

Scholarship@Western

Brain and Mind Institute Researchers'

Publications

Brain and Mind Institute

$9-1-2016$

\title{
Learning to be inflexible: Enhanced attentional biases in Parkinson's disease
}

Sean James Fallon

MRC Cognition and Brain Sciences Unit

Adam Hampshire

Imperial College London

Roger A. Barker

John van Geest Centre for Brain Repair

Adrian M. Owen

MRC Cognition and Brain Sciences Unit, uwocerc@uwo.ca

Follow this and additional works at: https://ir.lib.uwo.ca/brainpub

Citation of this paper:

Fallon, Sean James; Hampshire, Adam; Barker, Roger A.; and Owen, Adrian M., "Learning to be inflexible: Enhanced attentional biases in Parkinson's disease" (2016). Brain and Mind Institute Researchers' Publications. 981.

https://ir.lib.uwo.ca/brainpub/981 


\title{
Research report
}

\section{Learning to be inflexible: Enhanced attentional biases in Parkinson's disease}

\author{
Sean James Fallon ${ }^{a, b, *}$, Adam Hampshire ${ }^{c}$, Roger A. Barker ${ }^{d}$ and \\ Adrian M. Owen ${ }^{a, e}$ \\ ${ }^{a}$ Medical Research Council Cognition and Brain Sciences Unit, Cambridge, UK \\ b Department of Experimental Psychology, University of Oxford, Oxford, UK \\ c Computational Cognitive \& Clinical Neuroimaging Laboratory, Imperial College London, UK \\ ${ }^{\mathrm{d}}$ John van Geest Centre for Brain Repair, Department of Clinical Neurosciences, University of Cambridge, UK \\ e The Brain and Mind Institute, University of Western Ontario, CA, USA
}

\section{A R T I C L E I N F O}

Article history:

Received 9 December 2015

Reviewed 13 February 2016

Revised 12 April 2016

Accepted 12 May 2016

Action editor Sonja Kotz

Published online 24 May 2016

\section{Keywords:}

Parkinson's disease

Attention

Irrelevance

Set-shifting

Reward

\begin{abstract}
A B S T R A C T
Impaired attentional flexibility is considered to be one of the core cognitive deficits in Parkinson's disease (PD). However, the mechanisms that underlie this impairment are contested. Progress in resolving this dispute has also been hindered by the fact that cognitive deficits in PD are heterogeneous; therefore, it is unclear whether attentional impairments are only present in a subgroup of patients. Here, we demonstrate that what differentiates PD patients from age-matched controls is an inability to shift attention away from previously relevant information (perseveration) and an inability to shift attention towards previously irrelevant information (learned irrelevance). In contrast, there was no evidence that PD patients, compared to controls, were impaired in being able to appropriately attend to, or ignore, novel information. Furthermore, when patients were stratified according to their level of executive impairment, the executively impaired group showed a selective deficit in set formation compared to the unimpaired group, a behavioural pattern reminiscent of cortical dopamine depletion. Cumulatively, these results suggest that cognitive inflexibility in PD relates to a specific form of attentional dysfunction, in which learned attentional biases cannot be overcome.
\end{abstract}

(c) 2016 Elsevier Ltd. All rights reserved.

\section{Introduction}

Efficient functioning in the world requires that we are able to form and shift attentional sets (Fig. 1). Impaired attentional set-shifting is thought to constitute one of the core cognitive deficits found in Parkinson's disease (PD). Support for this claim comes from investigations that have utilised the Wisconsin Card Sorting Task (WCST) (Grant \& Berg, 1948) and analogue tests, and reported that PD patients are impaired in the ability to form and shift an attentional set and show aberrant frontostriatal processing (Downes et al., 1989; Gerrits

\footnotetext{
* Corresponding author. Department of Experimental Psychology, University of Oxford, OX13UD, UK.

E-mail address: sean.fallon@psy.ox.ac.uk (S.J. Fallon). 


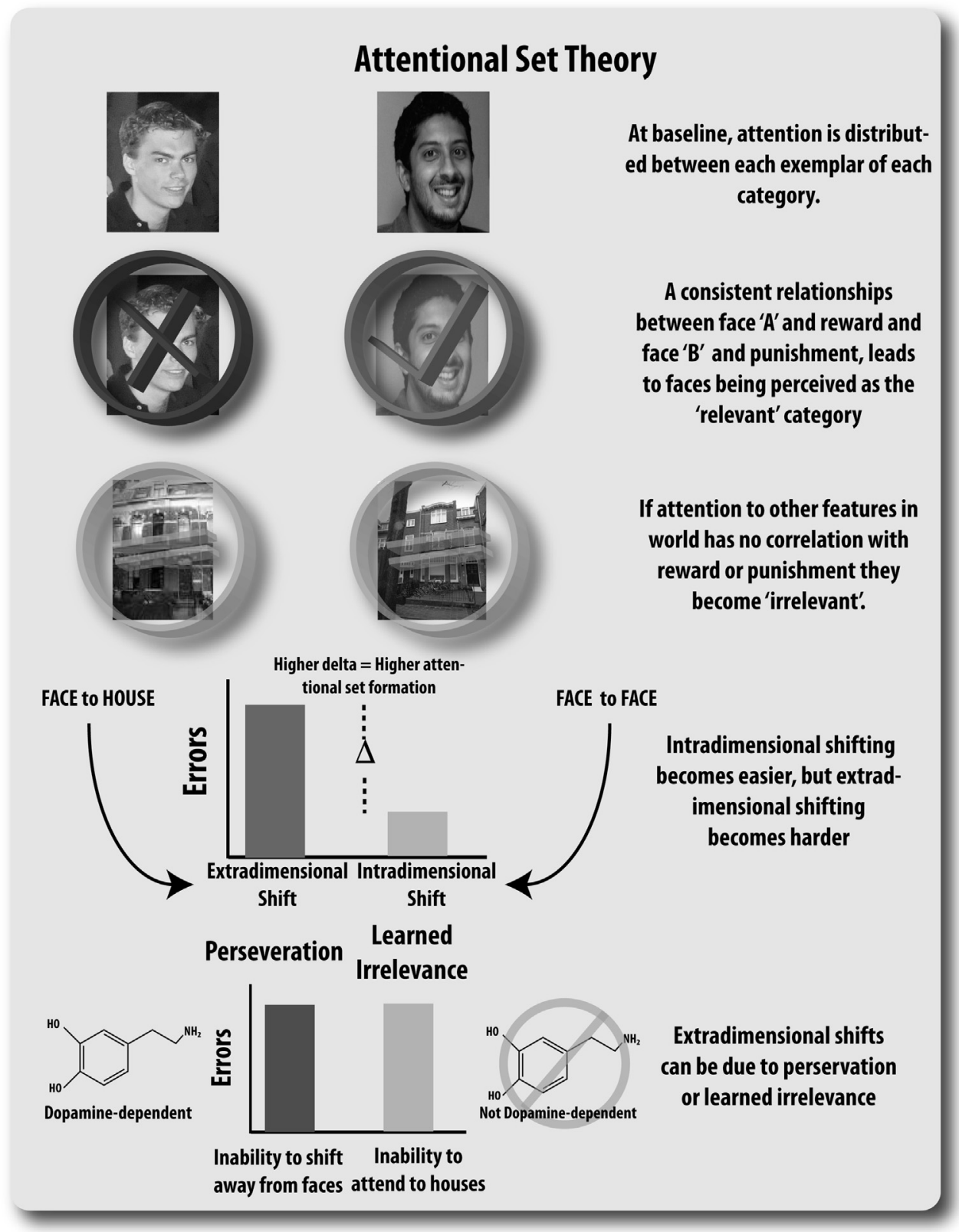

Fig. 1 - Attentional set theory describes how attentional allocation to certain features in the environment is established according to their correlation, or non-correlation with reward and punishment. Initially, there is no learned attentional bias towards one feature. However, if one face is consistently paired with reward and another face consistently paired with punishment, this means that attention towards faces is the crucial determinant in receiving either reward or punishment and therefore the dimension of 'faces' become the "relevant" dimension. In contrast, because there is no correlation between attention to houses and reward or punishment, the dimension of "houses" becomes irrelevant. This leads to the formation of an attentional set - the privileging of one class of information over another. The behavioural advantage of an attentional set is that it leads to a superior ability to switch attention within a dimension (face to face; intradimensional shift - IDS), but a relatively impaired ability to switch attention between different dimensions (face to house; extradimensional shift - EDS). However, extradimensional shifting is a heterogeneous process - it is comprised of preservation and learned irrelevance. Perseveration, in this context, is the inability or reluctance to shift attention away from previously relevant information, and is thought to dopamine-dependent. Learned irrelevance, however, is the inability or reluctance to attend to previously irrelevant information. Learned irrelevance is not thought to be dopamine-dependent.

et al., 2015; Harrison \& Owen, 2001; Monchi, Petrides, MejiaConstain, \& Strafella, 2007; Monchi et al., 2004; Moustafa, Sherman, \& Frank, 2008). Moreover, because most tasks in daily life involve focussing on some features whilst ignoring others, attentional sets provide the filter through which other higher-level cognitive functions operate. Thus, impaired attentional set formation and shifting, if present, are likely to have detrimental effects on patients' lives. However, our 
knowledge of the extent to which PD patients are impaired on attentional set-shifting tasks and the psychological mechanisms responsible for these deficits are limited and the subject of debate.

One hypothesis regarding the basis of PD patient's attentional set-shifting impairments is that learning makes their attention less flexible (Owen et al., 1993; Slabosz et al., 2006). For example, PD patients may have difficulty averting their attention from previously relevant information (perseveration) or because they cannot shift their attention to previously irrelevant information (learned irrelevance). In the context of attentional set-shifting tasks, learned irrelevance refers to the putatively reduced attention given to a perceptual dimension that has no correlation with reward (see Fig. 1). Learned irrelevance has been argued to be enhanced in patients with PD but is not affected by their dopaminergic status, unlike perseveration (Owen et al., 1993; Slabosz et al., 2006). Thus, these two impairments appear to have separate neural substrates, but can co-exist in PD patients.

Doubts have been raised, however, regarding the extent to which PD patients' behaviour on these tasks truly constitutes learned irrelevance (Cools, Rogers, Barker, \& Robbins, 2010; Gauntlett-Gilbert, Roberts, \& Brown, 1999; van Spaendonck, Berger, Horstink, Borm, \& Cools, 1995). Specifically, GauntlettGilbert et al. (1999) argued that PD patients do not have enhanced learned irrelevance (inability to attend to irrelevant information). A key prediction of the enhanced learned irrelevance account is that patients should become (relatively) more proficient with ignoring irrelevant information in all contexts. However, patients displayed equally poor levels of cognitive flexibility when either enhanced or reduced learned irrelevance was conducive to high-performance levels (Gauntlett-Gilbert et al., 1999), i.e., PD patients did not show the expected boost in performance that should come from being allowed to continue to ignore previously irrelevant information.

In addition, several studies found no difference between patients and controls in the level of learned irrelevance (Cools et al., 2010; van Spaendonck et al., 1995). These inconsistencies are likely to result from the use of betweensubject designs, which may produce null findings due to patient heterogeneity, i.e., certain cognitive deficits may only exist in certain sub-groups of patients (Habak et al., 2014; Hoogland et al., 2010; Nombela et al., 2014; Zokaei et al., 2014). Similarly, the ability of PD patients to form and shift attentional sets may vary according to differences in general executive functioning, i.e., attentional set-shifting deficits may only arise due to an impaired ability to effectively organise behaviour. Performance on the Tower of London, and related tasks, has been used to effectively stratify PD patients into those with and without executive impairment (Lewis, Cools, et al., 2003; Lewis, Dove, Robbins, Barker, \& Owen, 2003; Lewis et al., 2005). Such studies have found that the executively impaired group has abnormal working memory function with concomitant decreases in fronto-striatal activation (Lewis, Cools, et al., 2003; Lewis, Dove, et al., 2003). However, it remains to be determined whether a patient's level of executive dysfunction relates to a specific attentional impairment or a general (non-specific) reduction in performance.
This study sought to adjudicate between two hypotheses for impaired cognitive flexibility in PD patients: enhanced perseveration and learned irrelevance (Owen et al., 1993; Slabosz et al., 2006) or are impaired on all types of EDSs (Gauntlett-Gilbert et al., 1999). Furthermore, this study sought to examine whether patients' executive impairment (as indexed by Tower of London performance) modulated setshifting performance. This was achieved using a withinsubject design that allowed perseveration and learned irrelevance to be measured repeatedly.

We developed a novel computerised task in which participants were presented with four stimuli drawn from two different semantic categories. Like the closely-related WCST, after participants had reached a criterion they had to shift their attentional set. Here, however, instead of changing both categories, we measured learned irrelevance and perseveration by substituting either the previously relevant or previously irrelevant category with a novel category (Fig. 2B). This allowed for the separate quantification of learned irrelevance and perseveration. Crucially, we tested participants on conditions where learned irrelevance and perseveration were either adaptive or maladaptive, i.e., there were conditions where the previously relevant category had to be attended (perseveration succumb $\mathrm{P}+\mathrm{ve}$ ) to or ignored (perseveration overcome $\mathrm{P}-\mathrm{ve}$ ) and conditions where the previously irrelevant category had to be attended (overcome learned irrelevance LIRR-ve) or ignored (learned irrelevance succumb LIRR+ve). If PD patients really do display enhanced learned irrelevance then they should be relatively impaired when they have to attend to the previously irrelevant category (LIRR-ve), but relatively unimpaired when they can continue to ignore the previously irrelevant category (LIRR+ve). Similarly, if PD patients show enhanced perseveration they should be relatively impaired when they have to stop responding to the previously relevant category $(\mathrm{P}-\mathrm{ve})$ compared to succumbing to perseveration $(\mathrm{P}+\mathrm{ve})$. Alternatively, if $\mathrm{PD}$ patients are impaired in attentional set formation (Gauntlett-Gilbert et al., 1999) then patients should be impaired on extradimensional relative to IDSs.

\section{Method}

\subsection{Participants}

$40 \mathrm{PD}$ patients and 22 healthy older adults without a reported history of previous neurological complaints (e.g., stroke or head jury), psychiatric illness (as assessed using DSM-IV-TR diagnostic criteria for a psychiatric disorder), or visual impairment (e.g., colour blindness) were included in this study. Two patients who completed the task were excluded as they showed extremely repetitive behaviour - responding to the same stimuli during the course of the experiment (later quantified to be over 250 times in total). All patients met the United Kingdom Parkinson's Disease Society Brain Bank diagnostic criteria for PD. Only patients currently taking dopaminergic medications were included in this sample and all patients were tested on their usual anti-PD medication regime. A summary of the $\mathrm{PD}$ patients' medication regimes and clinical variables is presented in Supplementary Table 1. 


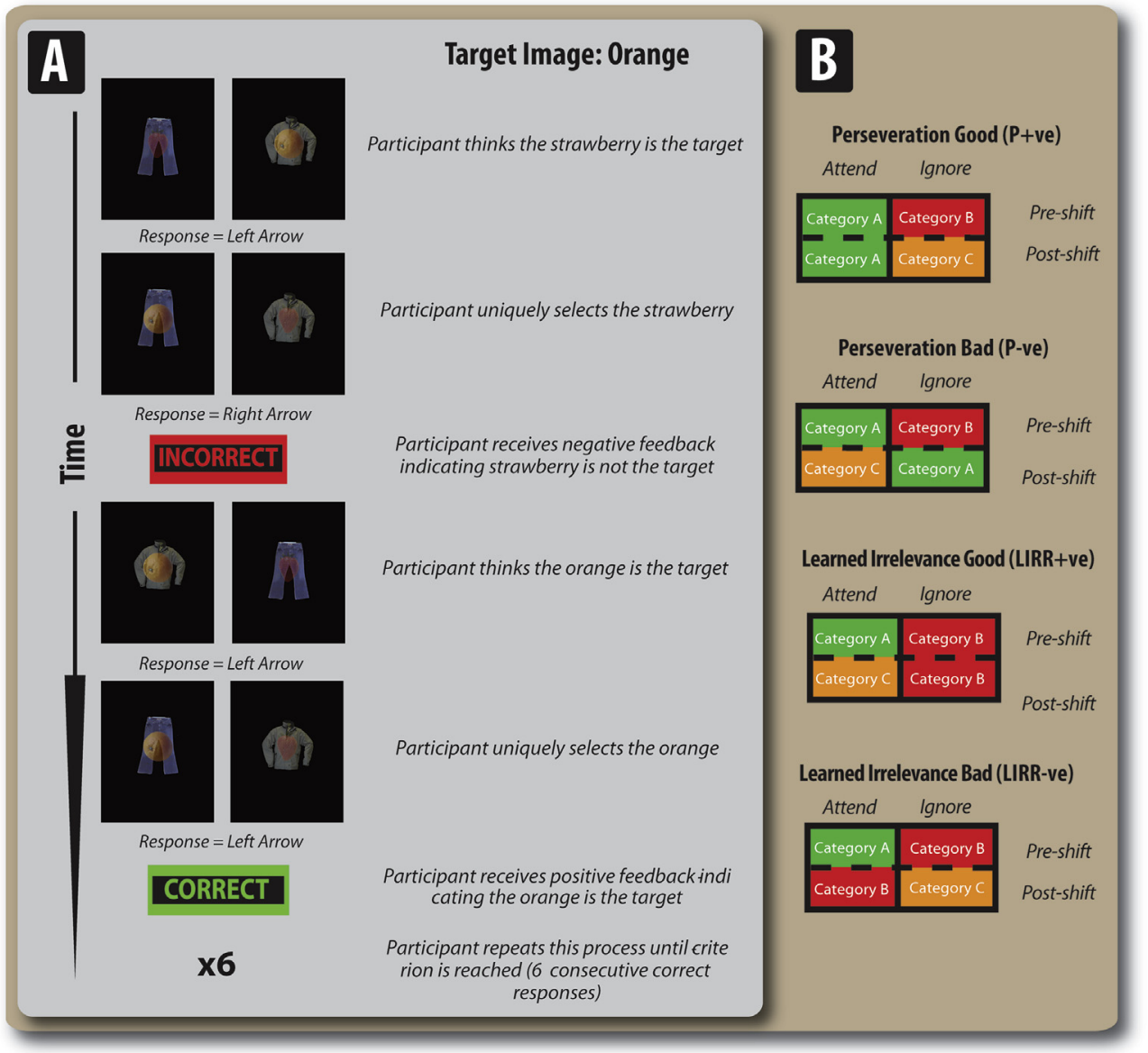

Fig. 2 - A) An example trial from the experiment. The participant's task was to work out, via trial and error, which, of the four objects presented on the screen, was the target image. They then had to continue to respond to this image until they had reached criterion (six responses; three feedback events). In this example, 'Fruit' is the relevant category because the target image is taken from this category, whereas 'clothes' is the irrelevant category because the target is not drawn from this category. Initially, the orange is chosen as the target, but, on subsequent trials, the answer will change (a reversal) to the strawberry. Thus, the participant 'explores' the relevant category by selectively attending to different exemplars drawn from the same category. Once participants reached a criterion, there was an extradimensional shift in which new exemplars appeared on the screen. The categories these exemplars were drawn from depended on the experimental condition. B) Illustration of the different types of extradimensional shifting. The key experimental manipulation in this study was to manipulate the presence/absence of the previous relevant/irrelevant categories and which category has to be attended to or ignored. For perseveration trials, the compound stimuli were made up of images drawn from the previously relevant category and a new novel category $(\mathrm{P}+\mathrm{ve}$ and $\mathrm{P}-\mathrm{ve})$. The two perseveration conditions were identical except that in $\mathbf{P}$ +ve the previously relevant category has to be attended to and in $\mathbf{P}$-ve it has to be ignored. For the learned irrelevance trials (LIRR+ve and LIRR-ve) the compound stimuli were made up of images from the previously irrelevant category and a new novel category. In the LIRR+ve trials, the previously irrelevant information had to be ignored, whereas in the LIRR-ve trials, the previously irrelevant information had to be attended to.

Healthy older adults were recruited from a local panel of volunteers. Patients and controls did not significantly differ in terms of age $[t(58)=.43, p=.66]$.

\subsection{Design and procedure}

\subsubsection{Set-shifting task}

The task was closely based on the CANTAB ID/ED task used previously (Owen et al., 1993). Participants were presented with a display that contained two compound images presented in panels to the left and the right of the screen (Fig. 2A). Each image was made up of two pictures superimposed onto each other, drawn from two separate categories, e.g., fruit \& houses. Participants had to discern, through trial-and-error, which of the four images currently presented on the screen was the target image. In these designs, the relevant category is the one that the target is drawn from, whereas the category from which the target is not drawn from is the irrelevant category (Fig. 2).

The procedure for each trial was identical to that in our previous studies (Hampshire \& Owen, 2006; Hampshire, Gruszka, Fallon, \& Owen, 2008). Participants could select one of the four images using the cursor keys on the keyboard 
(Fig. 2A). The left cursor was used to indicate an image in the left window and the right cursor was used to indicate an image from the right window. Participants could uniquely specify an image via two consecutive responses, because, on consecutive trials, each image was paired with an alternative exemplar from the other category. Thus, feedback was provided to participants after every second response. A green box at the centre of the screen, which contained the word "Correct", indicated a correct response whereas a red box at the centre of the screen that contained the word "Incorrect" indicated an incorrect response. After participants had found the target image, they then had to continue to select that image until they reached a criterion of six correct selections. After this, they would then move on to the next trial in the block (see Fig. 2A).

The task was made up of 20 blocks (comparable to 20 consecutive concatenated versions of the CANTAB ID/ED task). Each block consisted of five phases. Firstly, participants had to make discrimination within a dimension (initial trial), then shift responding to the other exemplar within this category (intradimensional reversal - IDR), generalise this discrimination to novel exemplars from the same category (IDS), then shift their attention towards a different category (EDS). Each block was identical until the EDS phase. Similar to (Gauntlett-Gilbert et al., 1999) and illustrated in Fig. 2B, there were four types of EDS: overcoming learned irrelevance (LIRR-ve), succumbing to learned irrelevance (LIRR+ve), overcoming Perseveration ( $\mathrm{P}-\mathrm{ve}$ ) and succumbing to perseveration $(\mathrm{P}+\mathrm{ve})$. The key experimental manipulation in this study was to manipulate the presence/absence of the previous relevant/irrelevant categories (see Fig. 2B). For example, on perseveration trials, the compound stimuli were made up of images drawn from the previously relevant category and a new novel category. This enabled the assessment of shifting to a new dimension, without the need to overcome the tendency to ignore the previously irrelevant dimension (learned irrelevance). For the learned irrelevance trials (LIRR-ve and LIRR+ve) the compound stimuli were made up of images from the previously irrelevant category and a new novel category the removal of the previously relevant category eliminated the need to inhibit responding to the previously relevant category (perseveration). Thus, only learned irrelevance could affect performance. However, PD patients could have deficits with overcoming perseveration or learned irrelevance simply because they are bad at performing the task. Therefore, following Gauntlett-Gilbert et al., 1999, we also included conditions were it would be beneficial to display learned irrelevance or perseveration ( $L I R R+v e$ and $\mathrm{P}+\mathrm{ve}$ trials). If $\mathrm{PD}$ patients do show enhanced learned irrelevance then they should show relatively enhanced performance on LIRR+ve trials relative to LIRR-ve trials.

To control for the possibility that asymmetries in the salience of each dimension would bias the results, there were two different sequences of stimuli used in this experiment. The categories and order of the two sequences can be found in the Supplementary materials (Supplementary Table 2). As can be seen from the table the two sequences were the mirror image of each other. Both sequences were divided into two runs. The order in which participants completed each run was counter-balanced. The side of the screen on which the correct image appeared was also counter-balanced to prevent the participant associating a given side with the correct exemplar.

\section{Stratification of patients into low and high executive functioning}

Performance on the "one-touch" Tower of London (Owen et al., 1995) task was used to stratify patients into high and low performing groups (Lewis, Cools, et al., 2003; Lewis, Dove, et al., 2003; Lewis et al., 2005). The Tower of London examines participants planning ability. Briefly, participants are presented with a display with two arrays on the top and bottom of the screen. Participants were required to mentally rearrange the balls on the bottom half of the display so as to match those in the top half (Goal state) and then respond, by touching the appropriate number on the bottom of the screen, to indicate how many "moves" this would take. Here, a median split on accuracy scores was used to separate the groups, with median scores removed from the analysis in order to increase power to detect a difference between the groups.

\subsection{Analysis}

Differences in dealing with relevant and irrelevant information in patients versus controls were assessed using mixed ANOVAs, where the main dependent variable was errors to criterion. All analyses were carried out using SPSS 22.0.

The first set of mixed ANOVAs examined the role of prior relevance or irrelevance and the need to overcome the previous attentional biases. Accordingly, the trial type (learned irrelevance or perseveration) and attentional bias (succumb or overcome) were entered as repeated measures. Disease (PD, controls) and cognitive impairment (unimpaired, impaired) were entered as between-subject factors to examine the effect of disease status and executive function, respectively.

Secondly, to control for the possibility that patients (or subgroups of patients) may have aberrant responses when attending to or ignoring novel information, we reformulated the above design matrix to compare trials where participants had to attend to novel information (LIRR+ve and $\mathrm{P}-\mathrm{ve}$ ) or ignore it (LIRR-ve and $\mathrm{P}+\mathrm{ve}$ ).

Thirdly, it has been claimed that PD patients do not show enhanced LIRR and instead show a deficit on all extradimensional, and not intradimensional, shifts (Gauntlett-Gilbert et al., 1999). Accordingly, to examine whether there is any evidence for this hypothesis the various types of shift employed in this study were directly compared in the exact same manner as previously (Gauntlett-Gilbert et al., 1999), i.e., compare EDSs (LIRR-ve, LIRR+ve, P-ve and) with intradimensional shifts (IDS, IDR, $\mathrm{P}+\mathrm{ve}$ ). Again, disease (PD, controls) and executive impairment (unimpaired, impaired) were entered as between-subject factors to examine the effect of disease status and executive function, respectively. Note that the $\mathrm{P}+\mathrm{ve}$ trials were designated as ID trials by (GauntlettGilbert et al., 1999), because, arguably, they do not involve any change in what participants have to attend to (see Fig. 2B). Thus, given that this represents the major alternative hypothesis to the learned irrelevance account, we chose to examine the hypothesis in exactly the same manner. 


\section{4. $\quad$ Results}

4.1. $\quad$ PD patients are impaired in overcoming perseveration and learned irrelevance

Errors for each shift type according to disease are shown in Fig. 3A. Disease had a significant main effect $[F(1,58)=5.53$, $p=.022$ ] on the number of errors as did the need to overcome previous attentional bias $[F(1,58)=19.98, p<.0001]$. There was also a significant two way interaction between disease and the need to overcome attentional biases on errors $[F(1,58)=4.30$, $p=.042]$. Simple main effects analyses revealed that this was due to PD patients making more errors than controls when perseveration or learned irrelevance needed to be overcome $[\mathrm{t}(58)=7.00, p=.010]$, whereas there was no difference between patients and controls when learned irrelevance and perseveration was allowed $[t(58)=1.64, p=.204]$. Thus, only when PD patients had to switch attention away from the previously

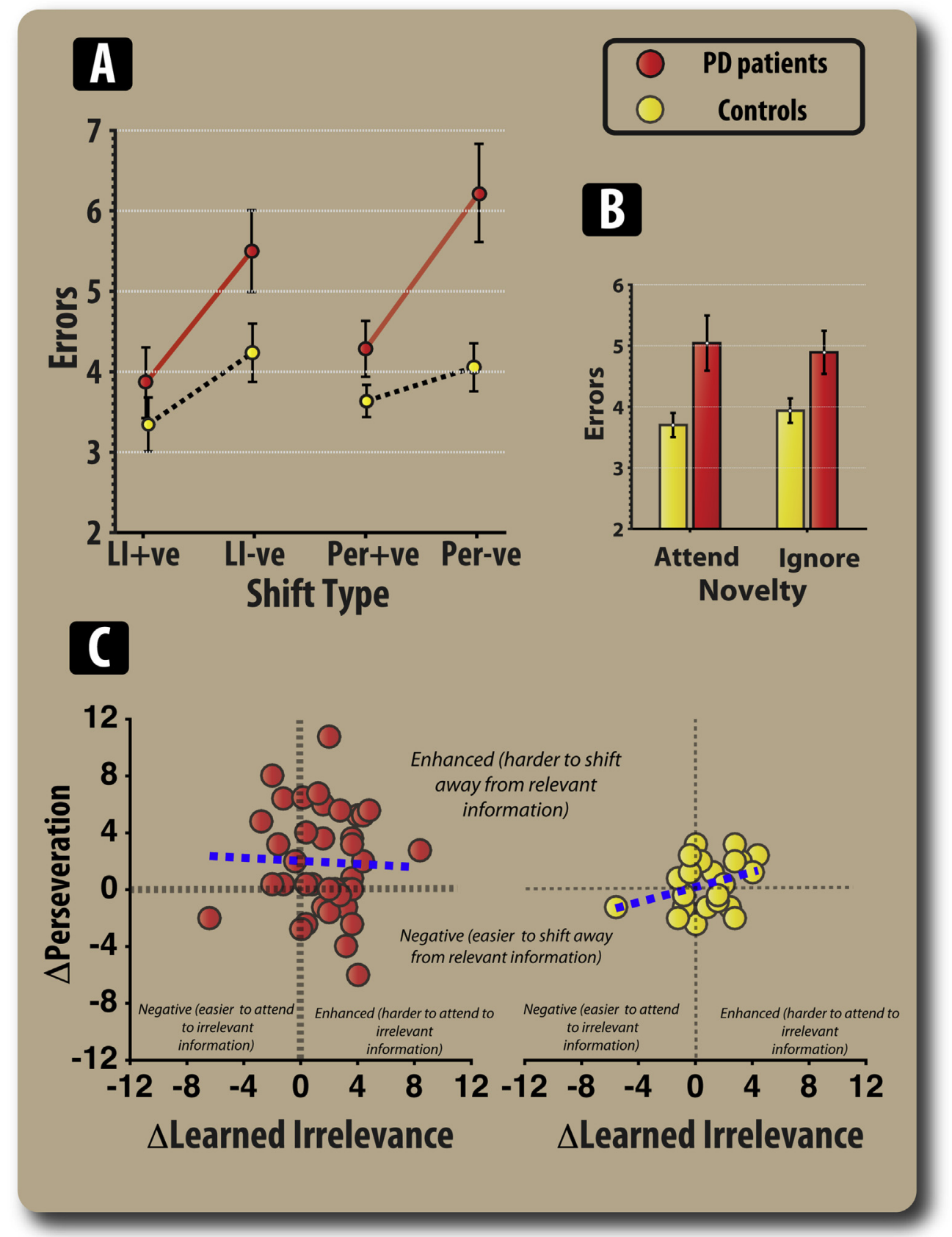

Fig. 3 - A) number of errors for patients and controls according to shift type. Error bars represent the standard error of the mean. B) Illustrating the effect of attending to novel (LIRR+ve and P-ve) and ignoring novel information (LIRR-ve and P+ve) in patients and controls C) Correlation between the level of learned irrelevance (LIRR-ve minus LIRR+ve) and the level of perseveration ( $\mathrm{P}-$ ve minus $\mathrm{P}+\mathrm{ve})$. The blue line represents the linear relationship (estimated using ordinary least squares) between the level of learned irrelevance and perseveration. 
relevant or attend to the previously irrelevant categories were they significantly impaired compared to controls.

We also reformulated our design matrix to examine whether PD patients had a specific deficit in attending to or ignoring novel information (Fig. 3B). There was a significant main effect of disease $[F(1,64)=5.53, p=.022]$ with patients making more errors overall. However, there was no significant main effect of having to attend to, or ignore, novel information nor any significant interaction with disease $(F s<1)$.

We also examined the hypotheses that PD patients had a specific deficit on extradimensional versus intradimensional shift errors (Gauntlett-Gilbert et al., 1999). These data are shown in Fig. 4A. There was a significant main effect of disease with patients making more errors than controls across both ID and ED shifts $[F(1,58)=5.88, p=.018]$. There was a significant main effect of shift type (ED vs ID) errors $[F(1,58)=4.4, p=.04]$, but no interaction with disease $[F(1,58)=1.77, p=.18]$. Thus, patients were impaired on both ID and ED shifts and showed no evidence of being differentially impaired in ID versus ED shifting. This confirmed that PD patients do not have a specific problem with shifting attention between different categories.

Given that the patients were on rather heterogeneous medication regimes (with some on dopamine agonists and others not), we sought to check whether this could affect our results. We therefore re-ran the above analyses, but only included those patients who were also taking dopamine agonists $(n=30)$. Restricting our analyses to this class of patients did not alter the interaction between disease and the need to overcome attentional basis $[F(1,50)=7.27, p=.010]$. Similarly, there was also no significant interaction between disease and shift type (ED us ID) errors $[F(1,50)=1.42, p=.239]$. Thus, different medication regimes cannot explain our above results. with each other, either within the whole sample $[r(60)=.06$, $p=.64]$ or within the PD group $[r(38)=-.03, p=.829$; Fig. $3 \mathrm{C}]$. Thus, although PD patients showed enhanced learned irrelevance and perseveration, the extent to which patients displayed either of these behaviours were not correlated with each other. Supplementary analyses examined the relationship between clinical variables (Unified Parkinson's disease rating scale - UPDRS, Beck Depression Inventory - BDI score, equivalent L-dopa and Hoehn and Yahr - H\&Y stage) and the above behaviours, but no significant relationships were found (Supplementary Table 4).

There was no difference between the executively impaired and unimpaired groups in terms of age, equivalent L-dopa dose, UPDRS score, BDI or H\&Y stage (Supplementary Table 3). We next examined whether the level of general executive impairment (performance on the Tower of London) could explain differential rates of learned irrelevance or perseveration in patients (Supplementary Fig. 1). There was a trend towards an interaction between executive functioning and learned irrelevance versus perseveration $[F(1,25)=3.16$, $p=.084]$. However, there was no main effect of executive impairment and none of the other interactions with executive impairment were significant ( $p$ 's $<.263$ ). Thus, level of executive impairment did not seem to produce robust differences between the display of learned irrelevance and perseveration.

In contrast, executive impairment significantly affected performance on extradimensional versus intradimensional shifts in PD patients $[F(1,25)=8.43, p=.008$; Fig. $4 B]$. Simple main effects analysis revealed that this was due to the lower cognitive function group showing no difference in extradimensional versus intradimensional shifting $[\mathrm{t}(25)=.32$, $p=.751$ ], whereas the high functioning PD group showed more ED versus ID errors $[\mathrm{t}(25)=3.84, p=.001]$.

\subsection{Individual differences in PD patients}

A measure of the strength of learned irrelevance (error on LIRR-ve minus LIRR+ve trials) and the strength of perseveration $(\mathrm{P}-\mathrm{ve}$ minus $\mathrm{P}+\mathrm{ve}$ ) showed no significant correlation

\section{Discussion}

This study has revealed that PD produces a difficulty in overcoming learned irrelevance and preservation. Thus

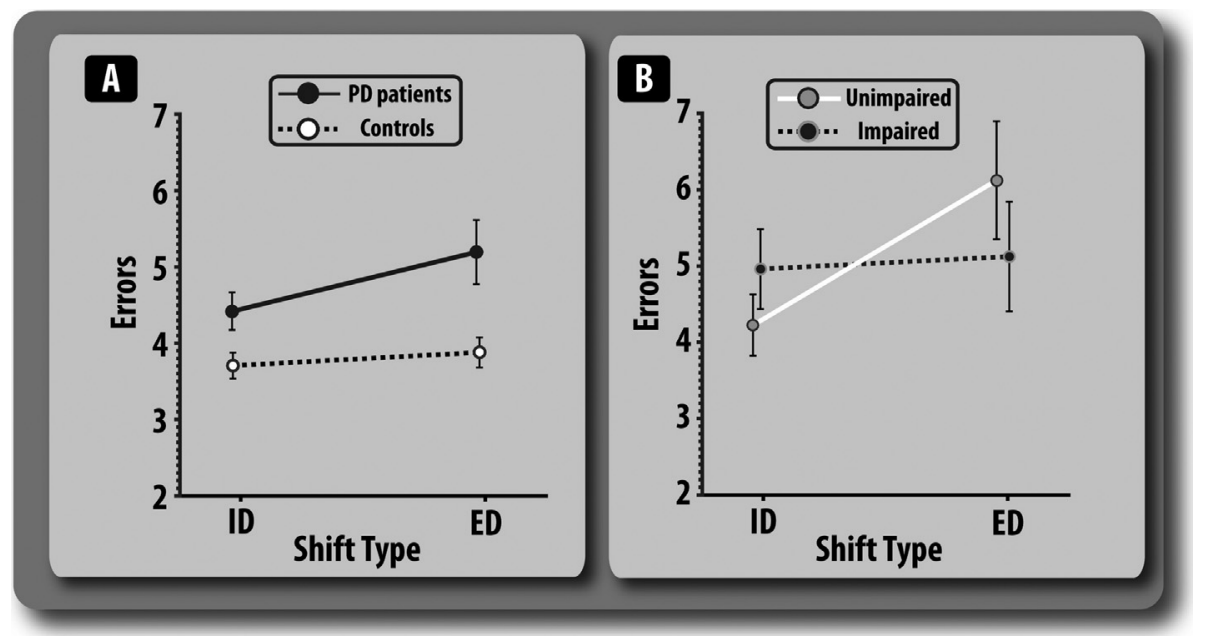

Fig. 4 - Number of errors on extradimensional (ED) and intradimensional (ID) shift trials for patients $(N=38)$ and controls (Panel A) and for a sub-groups of PD patients, split according to executive functioning level (panel B). Error bars represent the standard error of the mean. 
learning appears to make PD patients' attention less flexible. However, patients were not significantly impaired when either learned irrelevance or perseveration was conducive for good performance. Moreover, PD patients did not have a general deficit in re-orientating their attention as they were not disproportionately impaired in performing extradimensional compared to intradimensional shifts. PD patients were only impaired on certain types of EDSs - ones that involved attending to previously irrelevant information or shifting away from previously relevant information.

\subsection{Characterising attentional impairments in $\mathrm{PD}$}

These results suggest that the psychological mechanisms underlying impaired attentional control in PD patients involve an inability to disengage from previously relevant information and to re-engage with previously irrelevant information. The results of this study are consistent with previous reports (Owen et al., 1993; Slabosz et al., 2006). Importantly, this study was able to show, for the first time, that the ability of PD patients to set-shift can be relatively enhanced when it is useful to express learned irrelevance. Furthermore, previous studies have often confounded the assessment of learned irrelevance with the novelty of having to switch attention to other features in the environment. Thus, in previous studies any measure of learned irrelevance in PD patients was a mixture of a genuine problem in overcoming learned irrelevance and other undefined deficits in performing any cognitive operation for the first-time.

In contrast to the claim that $\mathrm{PD}$ patients have trouble overcoming learned irrelevance and perseveration, an alternative hypothesis has suggested that PD patients show a perceptual rigidity that makes shifting attention towards a new dimension harder than staying within the previously relevant dimension (Gauntlett-Gilbert et al., 1999), i.e., patients are impaired whenever there is any attentional reconfiguration in terms of what has to be attended to. This has been argued on the basis that previous studies have found that PD patients are equally impaired on all forms of EDSs (LIRR+ve, LIRR-ve and P-ve). This study found no evidence to support this hypothesis. Rather, as can be seen from Fig. 3A, the ability of PD patients to shift attention seems to be heavily structured by the prior relevance/irrelevance of the to-beattended-to dimension.

A related explanation for learned irrelevance is that patients have an aberrant response to novelty, i.e., patients find it difficult to attend or ignore novel information. However, as can be seen from Fig. 3B, there is no evidence for this. Thus, consistent with previous investigations, aberrant responses to novelty cannot account for increased learned irrelevance (Slabosz et al., 2006).

One area where the present findings may be thought to be in disagreement with previous findings (Owen et al., 1993), is that the patients in this study (who were medicated) showed enhanced perseveration, whereas medicated PD patients in the Owen et al. (1993) study did not show this. Furthermore, there was also no relationship between the level of medication (equivalent L-dopa dose) and the amount of perseveration. This could suggest that perseveration is not dopaminergically based. However, using equivalent L-dopa is an ersatz measure of patients' level of dopaminergic simulation as it is correlated with a variety of other factors such as disease severity. Therefore, future experiments will need to examine this further by directly manipulating or measuring dopamine levels in patients.

\subsection{Neural mechanisms behind enhanced learned irrelevance and perseveration in $\mathrm{PD}$}

This study found that there was no relationship between the level of perseveration and learned irrelevance (Fig. 3C). This is line with previous studies that have suggested that learned irrelevance and perseveration have separate neural substrates. Increased perseveration, unlike learned irrelevance, is thought to result from dopaminergic disruption and impaired frontal lobe functioning (Demakis, 2003; Egan et al., 2001; Malhotra et al., 2002; Meyer-Lindenberg et al., 2002; Owen et al., 1993; Takahashi et al., 2008). Enhanced learned irrelevance, in the context of attentional set-shifting, also appears to be something that is specific to PD [but see (Wong, Maybery, Bishop, Maley, \& Hallmayer, 2006)] and patients with striatal lesions (Swainson \& Robbins, 2001). Enhanced learned irrelevance has been found to be absent in numerous clinical conditions such as frontal lobe lesions (Owen et al., 1993), schizophrenia (Elliott, McKenna, Robbins, \& Sahakian, 1998; Elliott \& Sahakian, 1995), depression (Elliott et al., 1996), and Huntington's disease (Lawrence, Sahakian, Rogers, Hodge, \& Robbins, 1999). None of these clinical cohorts showed clear signs of having a deficit with overcoming learned irrelevance, despite being tested on exactly the same paradigm (Owen et al., 1993). More directly, recent functional neuroimaging studies have found increased striatal activation when switching attention to previously irrelevant information (LIRR-ve condition) than when continuing to ignore irrelevant information [LIRR+ve condition; see (Gruszka, Hampshire, \& Owen, 2010)]. The striatum is involved in processing rewarding information (Berns, McClure, Pagnoni, \& Montague, 2001; Delgado, Nystrom, Fissell, Noll, \& Fiez, 2000; Elliott, Newman, Longe, \& Deakin, 2003; Sescousse, Caldu, Segura, \& Dreher, 2013) and in propagating reward-related signals to cognitive and motoric networks (Aarts et al., 2010; Fallon \& Cools, 2014; Haber, Fudge, \& McFarland, 2000; Mogenson, Jones, \& Yim, 1980). Therefore, mechanistically, the striatum may be important for establishing that certain features in the world have no correlation with reward and are thus are deemed irrelevant (Fig. 1). Indeed, in an fMRI study, rewards were found to induce increases in the specific areas of cortex that are thought to be responsible for processing that category of sensory information, i.e., rewards presented after faces were found to elicit increased BOLD signal in the fusiform face area (Schiffer, Muller, Yeung, \& Waszak, 2014).

\subsection{The role of cognitive heterogeneity in structuring attentional impairments in PD}

One of the main behavioural advantages of attentional set formation - prioritising one type of information over another - is that it leads to an enhanced ability to shift within a dimension (IDS) and an impaired ability to shift between dimensions (EDS) (Roberts, Robbins, \& Everitt, 1988). Compared 
to controls, PD patients were not differentially impaired on extradimensional versus intradimensional shifts (Fig. 4). Altered set-formation was found to vary according to the level of executive impairment in patients. Previous studies have found that impaired executive functioning in PD patients - as assessed with the Tower of London - is associated with reductions in various nodes of the fronto-striatal network and working memory performance (Lewis, Cools, et al., 2003; Lewis, Dove, et al., 2003). Thus, it could have been hypothesised that uniform differences in performance may have arisen between the two groups. However, there was no overall difference in error rates. Rather, the two groups differed in terms of the distribution of errors on extradimensional compared to intradimensional shifts.

Reduced set-formation was found to occur in the executively impaired group compared to the unimpaired group. This pattern of performance in the executively impaired group is highly reminiscent of monkeys with 6-OHDA lesions in the frontal cortex, whereby reductions in cortical dopamine can abolish the difference between extradimensional and intradimensional shifting (Crofts et al., 2001; Roberts et al., 1994). Moreover, recent evidence suggests that deficits on set formation or EDSs only appear in sub-groups of PD patients according to their putative level of dopamine in the prefrontal cortex (Fallon, Hampshire, Williams-Gray, Barker, \& Owen, 2013; Fallon et al., 2015; Williams-Gray, Hampshire, Barker, \& Owen, 2008). Thus, at present, it is likely that the impaired group have reduced set-formation, presumably due to compromised dopaminergic functioning in the frontal lobe, although further studies will be needed to confirm this hypothesis. It is also interesting to note the performance pattern of healthy older adults, who performed equally well on intradimensional and EDSs, could also be said to support the lack of evidence for set formation. Given their performance levels were much higher than the patient group, it is possible that the task was too easy for them and thus did not require the formation of an attentional set, i.e., there was no need to trade off enhanced attention towards the relevant dimension at the expense of attentional flexibility (Fallon et al., 2013). Alternatively, because normal ageing is also associated with reductions in cortical dopamine levels (Backman, Nyberg, Lindenberger, Li, \& Farde, 2006; Kaasinen \& Rinne, 2002; Rieckmann et al., 2011), it may produce similar patterns of cognitive impairments as that which are seen after cortical damage and dopamine depletion (Barense, Fox, \& Baxter, 2002; Hampshire et al., 2008). Again, future studies should seek to alter or directly measure dopamine levels in healthy older adults and executively impaired PD patients in order to examine whether the aforementioned hypotheses are true.

\section{Summary}

This study sought to examine the mechanisms behind why PD patients find it hard to attend to feature $\mathrm{X}$ and ignore feature $\mathrm{Y}$ as this function is one of the foundations for higher-level cognition and adaptive behaviour. Within the form tested here, the results strongly suggest that deficits will only become apparent according to the prior relevance or irrelevance of features $\mathrm{X}$ and $\mathrm{Y}$. If feature $\mathrm{X}$ was previously irrelevant (learned irrelevance) then it will be harder to attend to, whereas if $\mathrm{Y}$ was previously relevant, it will be harder to ignore (perseveration). Furthermore, this study has shown that perseveration and learned irrelevance are separate psychological functions - possibly due to them having separate neural substrates - and co-exist in PD patients. Levels of executive performance (as measured by the Tower of London) were not related to the level of perseveration or learned irrelevance. However, it was related to the ability to form an attentional set, illustrating that although they perform at equivalent levels, the psychological mechanisms used to reach this are qualitatively different.

\section{Acknowledgements}

This research was supported by Medical Research Council (MRC) Grant (U1055.01.002.00001.01, AO). SJF was funded by a Medical Research Council studentship. In addition this work was also supported by grants from Medical Research Council (RG38582, RAB) and the Parkinson's Disease Society (RG39 906, $\mathrm{RAB})$, and the National Institutes of Health Research Biomedical Research Centre Award to the University of Cambridge and Addenbrooke's Hospital.

\section{Supplementary material}

Supplementary material related to this article can be found at http://dx.doi.org/10.1016/j.cortex.2016.05.005.

\section{R E F E R E N C E S}

Aarts, E., Roelofs, A., Franke, B., Rijpkema, M., Fernandez, G., Helmich, R. C., et al. (2010). Striatal dopamine mediates the interface between motivational and cognitive control in humans: evidence from genetic imaging. Neuropsychopharmacology, 35(9), 1943-1951. http://dx.doi.org/ 10.1038/npp.2010.68.

Backman, L., Nyberg, L., Lindenberger, U., Li, S. C., \& Farde, L. (2006). The correlative triad among aging, dopamine, and cognition: current status and future prospects. Neuroscience and Biobehavioral Reviews, 30(6), 791-807. http://dx.doi.org/ 10.1016/j.neubiorev.2006.06.005.

Barense, M. D., Fox, M. T., \& Baxter, M. G. (2002). Aged rats are impaired on an attentional set-shifting task sensitive to medial frontal cortex damage in young rats. Learning \& Memory, 9(4), 191-201. http://dx.doi.org/10.1101/lm.48602.

Berns, G. S., McClure, S. M., Pagnoni, G., \& Montague, P. R. (2001). Predictability modulates human brain response to reward. The Journal of Neuroscience, 21(8), 2793-2798. doi: 21/8/2793 [pii].

Cools, R., Rogers, R., Barker, R. A., \& Robbins, T. W. (2010). Topdown attentional control in Parkinson's disease: salient considerations. Journal of Cognitive Neuroscience, 22(5), 848-859. http://dx.doi.org/10.1162/jocn.2009.21227.

Crofts, H. S., Dalley, J. W., Collins, P., Van Denderen, J. C., Everitt, B. J., Robbins, T. W., et al. (2001). Differential effects of 6-OHDA lesions of the frontal cortex and caudate nucleus on the ability to acquire an attentional set. Cerebral Cortex, 11(11), 1015-1026. 
Delgado, M. R., Nystrom, L. E., Fissell, C., Noll, D. C., \& Fiez, J. A. (2000). Tracking the hemodynamic responses to reward and punishment in the striatum. Journal of Neurophysiology, 84(6), 3072-3077.

Demakis, G. J. (2003). A meta-analytic review of the sensitivity of the Wisconsin card sorting test to frontal and lateralized frontal brain damage. Neuropsychology, 17(2), 255-264.

Downes, J. J., Roberts, A. C., Sahakian, B. J., Evenden, J. L., Morris, R. G., \& Robbins, T. W. (1989). Impaired extradimensional shift performance in medicated and unmedicated Parkinson's disease: evidence for a specific attentional dysfunction. Neuropsychologia, 27(11-12), 1329-1343.

Egan, M. F., Goldberg, T. E., Kolachana, B. S., Callicott, J. H., Mazzanti, C. M., Straub, R. E., et al. (2001). Effect of COMT Val108/158 Met genotype on frontal lobe function and risk for schizophrenia. Proceedings of the National Academy of Sciences of the United States of America, 98(12), 6917-6922. http:// dx.doi.org/10.1073/pnas.111134598.

Elliott, R., McKenna, P. J., Robbins, T. W., \& Sahakian, B. I. (1998). Specific neuropsychological deficits in schizophrenic patients with preserved intellectual function. Cognitive Neuropsychiatry, 3(1), 45-69.

Elliott, R., Newman, J. L., Longe, O. A., \& Deakin, J. F. (2003). Differential response patterns in the striatum and orbitofrontal cortex to financial reward in humans: a parametric functional magnetic resonance imaging study. The Journal of Neuroscience, 23(1), 303-307. doi: 23/1/303 [pii].

Elliott, R., \& Sahakian, B. J. (1995). The neuropsychology of schizophrenia: relations with clinical and neurobiological dimensions. Psychological Medicine, 25(3), 581-594.

Elliott, R., Sahakian, B. J., McKay, A. P., Herrod, J. J., Robbins, T. W., \& Paykel, E. S. (1996). Neuropsychological impairments in unipolar depression: the influence of perceived failure on subsequent performance. Psychological Medicine, 26(5), 975-989.

Fallon, S. J., \& Cools, R. (2014). Reward acts on the pFC to enhance distractor resistance of working memory representations. Journal of Cognitive Neuroscience, 26(12), 2812-2826. http:// dx.doi.org/10.1162/jocn_a_00676.

Fallon, S. J., Hampshire, A., Williams-Gray, C. H., Barker, R. A., \& Owen, A. M. (2013). Putative cortical dopamine levels affect cortical recruitment during planning. Neuropsychologia, 51(11), 2194-2201. http://dx.doi.org/10.1016/ j.neuropsychologia.2013.07.016.

Fallon, S. J., Smulders, K., Esselink, R. A., van de Warrenburg, B. P., Bloem, B. R., \& Cools, R. (2015). Differential optimal dopamine levels for set-shifting and working memory in Parkinson's disease. Neuropsychologia, 77, 42-51. http://dx.doi.org/10.1016/ j.neuropsychologia.2015.07.031.

Gauntlett-Gilbert, J., Roberts, R. C., \& Brown, V. J. (1999). Mechanisms underlying attentional set-shifting in Parkinson's disease. Neuropsychologia, 37(5), 605-616.

Gerrits, N. J., van der Werf, Y. D., Verhoef, K. M., Veltman, D. J., Groenewegen, H. J., Berendse, H. W., et al. (2015). Compensatory fronto-parietal hyperactivation during setshifting in unmedicated patients with Parkinson's disease. Neuropsychologia, 68, 107-116. http://dx.doi.org/10.1016/ j.neuropsychologia.2014.12.022.

Grant, D. A., \& Berg, E. A. (1948). A behavioral analysis of degree of reinforcement and ease of shifting to new responses in a weigl-type card-sorting problem. Journal of Experimental Psychology, 38(4), 404-411. http://dx.doi.org/10.1037/H0059831.

Gruszka, A., Hampshire, A., \& Owen, A. M. (2010). Learned irrelevance revisited: pathology-based individual differences, normal variation and neural correlates. Handbook of Individual Differences in Cognition, 127-144.
Habak, C., Noreau, A., Nagano-Saito, A., Mejia-Constain, B., Degroot, C., Strafella, A. P., et al. (2014). Dopamine transporter SLC6A3 genotype affects cortico-striatal activity of set-shifts in Parkinson's disease. Brain, 137(Pt 11), 3025-3035. http:// dx.doi.org/10.1093/brain/awu251.

Haber, S. N., Fudge, J. L., \& McFarland, N. R. (2000). Striatonigrostriatal pathways in primates form an ascending spiral from the shell to the dorsolateral striatum. The Journal of Neuroscience, 20(6), 2369-2382.

Hampshire, A., Gruszka, A., Fallon, S. J., \& Owen, A. M. (2008). Inefficiency in self-organized attentional switching in the normal aging population is associated with decreased activity in the ventrolateral prefrontal cortex. Journal of Cognitive Neuroscience, 20(9), 1670-1686. http://dx.doi.org/10.1162/ jocn.2008.20115.

Hampshire, A., \& Owen, A. M. (2006). Fractionating attentional control using event-related fMRI. Cerebral Cortex, 16(12), 1679-1689. http://dx.doi.org/10.1093/cercor/bhj116.

Harrison, J. E., \& Owen, A. M. (2001). Research issues in the neuropsychological investigation of brain disease. Cognitive Deficits in Brain Disorders, 1.

Hoogland, J., de Bie, R. M., Williams-Gray, C. H., Muslimovic, D., Schmand, B., \& Post, B. (2010). Catechol-O-methyltransferase val158met and cognitive function in Parkinson's disease. Movement Disorders, 25(15), 2550-2554. http://dx.doi.org/ 10.1002/mds.23319.

Kaasinen, V., \& Rinne, J. O. (2002). Functional imaging studies of dopamine system and cognition in normal aging and Parkinson's disease. Neuroscience and Biobehavioral Reviews, 26(7), 785-793.

Lawrence, A. D., Sahakian, B. J., Rogers, R. D., Hodge, J. R., \& Robbins, T. W. (1999). Discrimination, reversal, and shift learning in Huntington's disease: mechanisms of impaired response selection. Neuropsychologia, 37(12), 1359-1374.

Lewis, S. J., Cools, R., Robbins, T. W., Dove, A., Barker, R. A., \& Owen, A. M. (2003). Using executive heterogeneity to explore the nature of working memory deficits in Parkinson's disease. Neuropsychologia, 41(6), 645-654.

Lewis, S. J., Dove, A., Robbins, T. W., Barker, R. A., \& Owen, A. M. (2003). Cognitive impairments in early Parkinson's disease are accompanied by reductions in activity in frontostriatal neural circuitry. The Journal of Neuroscience, 23(15), 6351-6356.

Lewis, S. J., Foltynie, T., Blackwell, A. D., Robbins, T. W., Owen, A. M., \& Barker, R. A. (2005). Heterogeneity of Parkinson's disease in the early clinical stages using a data driven approach. Journal of Neurology, Neurosurgery, and Psychiatry, 76(3), 343-348. http://dx.doi.org/10.1136/ jnnp.2003.033530.

Malhotra, A. K., Kestler, L. J., Mazzanti, C., Bates, J. A., Goldberg, T., \& Goldman, D. (2002). A functional polymorphism in the COMT gene and performance on a test of prefrontal cognition. The American Journal of Psychiatry, 159(4), $652-654$.

Meyer-Lindenberg, A., Miletich, R. S., Kohn, P. D., Esposito, G., Carson, R. E., Quarantelli, M., et al. (2002). Reduced prefrontal activity predicts exaggerated striatal dopaminergic function in schizophrenia. Nature Neuroscience, 5(3), 267-271. http:// dx.doi.org/10.1038/nn804.

Mogenson, G. J., Jones, D. L., \& Yim, C. Y. (1980). From motivation to action: functional interface between the limbic system and the motor system. Progress in Neurobiology, 14(2-3), 69-97.

Monchi, O., Petrides, M., Doyon, J., Postuma, R. B., Worsley, K., \& Dagher, A. (2004). Neural bases of set-shifting deficits in Parkinson's disease. The Journal of Neuroscience, 24(3), 702-710. http://dx.doi.org/10.1523/JNEUROSCI.4860-03.2004.

Monchi, O., Petrides, M., Mejia-Constain, B., \& Strafella, A. P. (2007). Cortical activity in Parkinson's disease during executive 
processing depends on striatal involvement. Brain, 130(Pt 1), 233-244. http://dx.doi.org/10.1093/brain/awl326.

Moustafa, A. A., Sherman, S. J., \& Frank, M. J. (2008). A dopaminergic basis for working memory, learning and attentional shifting in Parkinsonism. Neuropsychologia, 46(13), 3144-3156. http://dx.doi.org/10.1016/ j.neuropsychologia.2008.07.011.

Nombela, C., Rowe, J. B., Winder-Rhodes, S. E., Hampshire, A., Owen, A. M., Breen, D. P., et al. (2014). Genetic impact on cognition and brain function in newly diagnosed Parkinson's disease: ICICLE-PD study. Brain, 137(Pt 10), 2743-2758. http:// dx.doi.org/10.1093/brain/awu201.

Owen, A. M., Roberts, A. C., Hodges, J. R., Summers, B. A., Polkey, C. E., \& Robbins, T. W. (1993). Contrasting mechanisms of impaired attentional set-shifting in patients with frontal lobe damage or Parkinson's disease. Brain, 116(Pt 5), 1159-1175.

Owen, A. M., Sahakian, B. J., Hodges, J. R., Summers, B. A., Polkey, C. E., \& Robbins, T. W. (1995). Dopamine-dependent frontostriatal planning deficits in early Parkinson's disease. Neuropsychology, 9(1), 126.

Rieckmann, A., Karlsson, S., Karlsson, P., Brehmer, Y., Fischer, H., Farde, L., et al. (2011). Dopamine D1 receptor associations within and between dopaminergic pathways in younger and elderly adults: links to cognitive performance. Cerebral Cortex, 21(9), 2023-2032. http://dx.doi.org/10.1093/cercor/bhq266.

Roberts, A. C., De Salvia, M. A., Wilkinson, L. S., Collins, P., Muir, J. L., Everitt, B. J., et al. (1994). 6-Hydroxydopamine lesions of the prefrontal cortex in monkeys enhance performance on an analog of the Wisconsin Card Sort Test: possible interactions with subcortical dopamine. The Journal of Neuroscience, 14(5 Pt 1), 2531-2544.

Roberts, A. C., Robbins, T. W., \& Everitt, B. J. (1988). The effects of intradimensional and extradimensional shifts on visual discrimination learning in humans and non-human primates. The Quarterly Journal of Experimental Psychology B, 40(4), 321-341.

Schiffer, A. M., Muller, T., Yeung, N., \& Waszak, F. (2014). Reward activates stimulus-specific and task-dependent representations in visual association cortices. The Journal of Neuroscience, 34(47), 15610-15620. http://dx.doi.org/10.1523/ JNEUROSCI.1640-14.2014.

Sescousse, G., Caldu, X., Segura, B., \& Dreher, J. C. (2013). Processing of primary and secondary rewards: a quantitative meta-analysis and review of human functional neuroimaging studies. Neuroscience and Biobehavioral Reviews, 37(4), 681-696. http://dx.doi.org/10.1016/j.neubiorev.2013.02.002S01497634(13)00037-7 [pii].

Slabosz, A., Lewis, S. J. G., Smigasiewicz, K., Szymura, B., Barker, R. A., \& Owen, A. M. (2006). The role of learned irrelevance in attentional set-shifting impairments in Parkinson's disease. Neuropsychology, 20(5), 578-588.

van Spaendonck, K. P., Berger, H. J., Horstink, M. W., Borm, G. F., \& Cools, A. R. (1995). Card sorting performance in Parkinson's disease: a comparison between acquisition and shifting performance. Journal of Clinical and Experimental Neuropsychology, 17(6), 918-925.

Swainson, R., \& Robbins, T. W. (2001). Rule-abstraction deficits following a basal ganglia lesion. Neurocase, 7(5), 433-443. http://dx.doi.org/10.1076/neur.7.5.433.16248.

Takahashi, H., Kato, M., Takano, H., Arakawa, R., Okumura, M., Otsuka, T., et al. (2008). Differential contributions of prefrontal and hippocampal dopamine $\mathrm{D}(1)$ and $\mathrm{D}(2)$ receptors in human cognitive functions. The Journal of Neuroscience, 28(46), 12032-12038. http://dx.doi.org/10.1523/JNEUROSCI.344608.2008 .

Williams-Gray, C. H., Hampshire, A., Barker, R. A., \& Owen, A. M. (2008). Attentional control in Parkinson's disease is dependent on COMT val 158 met genotype. Brain, 131(Pt 2), 397-408. http://dx.doi.org/10.1093/brain/awm313.

Wong, D., Maybery, M., Bishop, D. V., Maley, A., \& Hallmayer, J. (2006). Profiles of executive function in parents and siblings of individuals with autism spectrum disorders. Genes, Brain and Behavior, 5(8), 561-576.

Zokaei, N., McNeill, A., Proukakis, C., Beavan, M., Jarman, P., Korlipara, P., et al. (2014). Visual short-term memory deficits associated with GBA mutation and Parkinson's disease. Brain, 137(Pt 8), 2303-2311. http://dx.doi.org/10.1093/brain/awu143. 